Shaban I, Barclay LM, Lock L, Homer CSE. (2012) Barriers to developing midwifery as a primary health care strategy: A Jordanian study. Midwifery 28(1):10611.

\title{
Barriers to developing midwifery as a primary health care strategy: A Jordanian study
}

\section{Background}

A primary health care strategy emphasises working with people to enable them to make decisions about their needs and how best to address them (Wass, 2000). Since the World Health Organization (WHO) adopted a primary health care strategy more than 30 years ago, primary health care has made an important contribution to achieving improvements in health in many countries (Briggs et al., 2005), including Jordan. The World Health Organization sought to achieve health for all by changing the focus of a nation's health system infrastructure to provide primary health care that reached the whole population in national programs. This was seen as the key to attaining Health for All (WHO, 1978). Primary health care is a fundamental part of midwifery practice (Shah and King, 2006; Homer et al, 2007).

Primary health care within a public health strategy focuses on improving the health of the population. This ensures that the right care is provided to the right people, by the most appropriate provider, in the right setting using the most suitable and costeffective technology (Briggs et al., 2005). In line with this, an analysis of policy and legal barriers to improve reproductive health services was undertaken by the Jordan National Population Commission (2000, p. 33). The Commission reported that the removal of barriers to effective use of reproductive primary health care services could be achieved by: "Full utilisation of midwives to provide female care for female clients and to improve overstretched clinical and counselling services”.

There is agreement in Jordan that a midwife is the most suitable person to be assigned to the care of women as a female of the relevant culture, religion, and gender. PetroNustas (2001, p. 191) identified that "women are reluctant to be examined by male providers, which increases demand for care to be provided by women. For those women, who observe the traditional rules of modesty, a female community health 
nurse or midwife may be a better alternative than general practitioner or obstetricians as most are male". Despite this, midwives have not been well utilised in Jordan (Jordan National Population Commission, 2000).

In Jordan, antenatal and postnatal care is provided in Primary Health Care (PHC) centres with labour and birth managed in hospitals. The antenatal care provider is a physician for $92 \%$ of women in urban areas and 83\% in rural regions (JPFHS, 2002). Antenatal care includes blood pressure measurement, weight at every visit and a haemoglobin check at the first visit. The urine is tested at least once during the pregnancy, the fetal heart rate is measured at each visit, and prescription of iron, folate and tetanus toxoid occurs for all pregnant women. There is no evidence that counselling to determine women's needs and demands is viewed as a core component of care. Although most births (98\%) are attended by trained health personnel (WHO, 2002), midwives usually assist obstetricians, perform procedures (for example, vaginal examinations, episiotomies) and assume responsibilities for the care of lowrisk women. Obstetricians, however, are the primary decision-makers and judge the need to use any drugs, including analgesia (Abushaikha \& Oweis, 2005). The practice of midwives in hospitals, is fragmented in specific areas, such as antenatal ward, labour ward or the postnatal ward, means their practice. Women who have a normal birth are usually discharged within 24 hours. The physician gives women an appointment for follow-up four weeks after discharge. Only one quarter of women receive additional postnatal care (Pedersen \& Hanssen-Bauer, 1998). Primarily, physicians, in a single consultation, provide this care in a postnatal visit. There is little follow-up in the immediate postnatal period and no home visits.

Recognising that midwives should be utilised fully in the provision of reproductive primary health care and family-planning services in Jordan is an important step forward. It would be helpful in arguing the case if the perceptions of the midwives about working in a primary health care strategy were known. The aims of the study were to explore the perceptions and attitudes of midwifery clinicians and educators in relation to midwifery as a primary health care strategy, and to understand the barriers for midwives to undertake this role. The study sought to answer two research questions: 
1. What are the barriers to developing midwifery as a primary health care strategy in Jordan?

2. What are the strategies to overcome these barriers?

The findings of the study will inform policy-makers, institutions and educators on the educational programs that are needed for the future. This could make a difference to the health status of the community and region if changes are made that are consistent with primary health care policy and meet the cultural and spiritual needs of Moslem women. There are no previous studies that have been conducted to better understand the potential utility of midwives in Jordan.

\section{Methodology}

An exploratory design using an action research approach was undertaken. This study used the principles of participatory-research engagement as a form of action research (Cornwall and Jewkes, 1995). Action research is a form of self-reflective inquiry undertaken by participants in social situations in order to justify their own social practices, as well as their understanding of the practices and situation in which these practices are carried out (Carr and Kemmis, 1986; Meyer, 1993; Noffke and Stevenson, 1995). Action research is not limited to the collection of qualitative data and can use a variety of complementary methods to produce a rich picture of events from the perspective of those who experience them (Cornwall and Jewkes, 1995).

The goal of action research is participation as a goal in itself and this can be expressed in terms of empowering people. The key methodological feature that distinguishes action research from other social research is dialogue (Sohng, 1995). Hughes et al. (1994) identified that a successful plan for action research must include a statement of the aim for change or improvement and identification of the knowledge to be generated. According to these authors, action research is used when the outcome has been identified but it is not known how to achieve it (Hughes et al., 1994). This is similar to the situation in Jordan, as the government wants to improve midwifery as a profession but does not know how to do this. Action research was an appropriate methodology as it brought together isolated and widely distributed people together 
around common problems; validated their experiences; and presented the knowledge and experiences of the researcher as additional resources upon which to reflect upon (Sleeples, 2004) as solutions to the problem were identified.

We aimed to stimulate thought and possible change in midwifery and maternity care through processes of self-reflection and human interaction. This was needed before action or change could occur. Those affected by the situation needed to first understand that which was occurring. The first author worked as a 'consultant researcher' with practising midwives and educators, to help them consolidate their opinions and expectations about their role within a primary health care strategy. The study was conceptualized and designed with the other authors who assisted with the writing and data analysis.

\section{Ethics approval}

The research proposal was submitted to the Ministry of Health (MOH) and approved. Approval for the study was also obtained from the Human Research Ethics Committee of the University of Technology, Sydney Australia. For each midwife recruited; researchers provided written and oral information about the study. Informed consent was obtained from each participant by means of a signed form. Participants were informed that their participation was voluntary, and they could withdraw at any time.

\section{Sample}

Midwives who were employed in Jordanian government hospitals and Maternal and Child Health (MCH) centres were invited to participate. An opportunistic sample was drawn from educators and clinicians across the three regions of Jordan (north, south, central). Permission to approach midwives was given by the Assistant, Secretary General for PHC; Assistant, Secretary General for Hospitals; and, the Director of Manpower Development. Midwives in each region from education and service sectors were approached. Both sectors were included because educators are involved in clinical training of midwifery students. Educators were from both nursing and midwifery government colleges (there are only two) and the two universities that teach midwifery and reproductive health. The manager for each of the government 
hospitals and centres provided local approval and support. One manager refused to send the required number of midwives, due to staff shortages and high workload.

The place and time of the workshop were confirmed by telephone. Workshops were conducted in English and Arabic. The characteristics and numbers of staff who participated are summarised in Table 1. Initially, experienced midwives were approached because appropriate experience and homogeneity is usually the key consideration in selecting respondents (Hoskins and Mariano, 2004; Polit and Beck, 2004).

The process of selecting participants into the workshops took place in collaboration with the managers. The purpose of the study aimed to obtain more in-depth information than could have been possible in a design that included more participants. An important part of the study was the provision of information as well as generation of new knowledge.

\section{Data collection}

Data collection occurred during the workshops. The workshop began with introductions and welcoming of participants. Participants were provided with an overview of the agenda including the information letter, consent form and demographic details to complete and finished by a presentation that was used initially to stimulate discussion. The first session obtained background information on the participants, and familiarised them with the issues of seeing midwifery within a PHC strategy and how midwives could undertake this role. The presentation was based on an analysis of the literature and a number of policy documents (Policy Project, 2000a, 2000b, 2000c) and covered the following guiding principles and open-ended questions:

- If pregnancy and childbirth are normal and significant life events why are maternity services designed the way they are?

- The right of women to be the focus of maternity care. What is the woman's capacity to be in control and make informed decision about her care? Is this 
care based on her needs? What is her right to culturally and spiritually appropriate care?

- Internationally, midwifery care follows the woman across the interface between institutions and the community, through all phases of pregnancy, birth and the postnatal period. It involves collaboration with other health professionals. How could this interface be improved?

- If evidence shows midwifery care can be provided in a woman's home, hospital and PHC centre settings, what models could work in Jordan?

These questions were triggers for discussion and not piloted or tested outside the group of authors who are experienced in generating information from interviews and focus groups.

In the second session, participants were split into four small groups to discuss and explore the barriers that prevent midwives working as primary health care providers and to identify the strategies to overcome these barriers. Each group was asked to write notes on flip charts and present an overview of their discussion to the whole group. Participants choose to talk in either language. As both facilitators are fluent in both languages this was not a major concern. Each workshop lasted four hours. The first author led the workshops and the second author assisted with facilitating the group with the other author taking notes of what was said. The tape-recordings of the workshops were transcribed verbatim and translated into English.

\section{Data analysis}

Participants were asked to reflect on the problems discussed. The researcher requested that they verbalise their thoughts and considerations while they answered the questions (Stringer, 1999) The transcribed audio-tape recordings were analysed for themes. Themes were extracted by one group and then the first author repeated these to the wider group as a means of validation. Themes were then analysed within the policy and educational context in Jordan. The analysis conducted after each workshop guided the next workshop which produced an ongoing and developing dialogue informed by participants that was confirmed or further developed in the next group. 
Participants described their role and practice as being constrained by several factors grouped around service provision and the practice domain of midwives. The main emergent themes are grouped under five headings using quotations from groups of participants to illustrate these themes. The actual words spoken by participants have been identified using inverted commas.

\section{Results}

A total of 64 midwives from the 70 (91\%) who were invited participated in the workshops participated. This included 14 working in a PHC services and 12 working as midwifery educators. The mean age of the 64 midwives in the workshops was 35 years, with a range from 30-39 years (32/64, 50\%). Most were married (37/64, 58\%) with at least eight years' experience in midwifery (33/64, 52\%). Most had a graduate diploma in midwifery qualification (54/64, 84\%), and the majority of them worked in hospitals (36/64, 56\%). Most clinicians experienced highly interventionist and medicalised maternity care where the doctor and the hospital directed the process. In addition to that, midwives provided fragmented care with a lack of continuity of care

The five main barriers identified were a lack of professional recognition of midwifery; a lack of recognition and status of midwifery within society; individual occupational stress and a high workload; the medical domination of the health system; and, issues to do with midwifery education. These barriers and corresponding strategies are presented in the next section.

\section{Professional recognition}

Reports of lack of professional recognition of midwifery and lack of understanding of the role of the midwife came equally strongly from midwives in service provision as from education. It was widely felt that the invisibility of midwifery as a profession was one of the most serious barriers to improved usage of midwives as primary health care providers. For some, this occurred because of a lack of recognition of the benefits of midwifery and the high level of nursing and medical dominance of midwifery practice that restricted midwives' desire to fulfil their role. One group of practising midwives said: 
"We need to look for the policies that determine the role of the midwife, where it lies in the health team" (practising midwives - Group one).

Midwifery is not recognised as a separate profession in Jordan, therefore midwifery managers have been downgraded and nurses manage midwives. This has affected the confidence of the midwife in her own abilities and has influenced how she works. Participants described an urgent need for role models a skilled midwifery leaders. This need was exacerbated by an overt focus on 'nursing' in matters of professional education, management and organisational leadership

\section{Societal recognition and image}

The lack of recognition and status of midwifery within society was identified as a barrier to midwifery's ability to make a significant contribution to improving health outcomes. This social image was contributed to by the lower entry criteria to midwifery compared with nursing, as well as to any other comparable professions. Midwifery historically has been the cheapest program offered through the Ministry of Health and has been devalued. The societal image is also influenced by consumers' emphasis on technology and the belief that this is better than dealing with birth as a natural event.

Participants identified need for greater understanding about midwifery within the community. It was argued that education and marketing had to be improved to inform women fully about the role that midwives could play in their reproductive health and to assist them to make informed choices. Strategies were suggested including promoting public awareness and educating local doctors about the role and functions of midwives. One of the practising midwives and midwifery educators illustrated this by saying:

\footnotetext{
"There is an absence of any public education about midwives and keeping birth normal this is now becoming an urgent public health issue” (practising midwives - Group Two).
}

"It [education programs] must target the women themselves and talk about what [a] midwife can do under her own responsibility. That would change the image of the midwife" (midwifery educators). 
Participants considered that education about midwifery, for both medical personnel and the public, was one of the most important parts of the implementing improvement in maintaining and updating regulated midwifery in Jordan.

\section{Stress and workload}

In identifying their concerns about the quality and outcomes of care within maternity units, some participants detailed the consequences of working in stressful environments. They identified lack of time for providing 'non-medical' or 'nonurgent care’. For example:

"Management has completely unrealistic expectations regarding financial goals versus delivery of safe care. [The] ratio of midwife to patient is 1:20 or more. We cannot do the tasks required even for safe practice. In addition to that, the pregnancy record and family planning record makes considerable work for midwives, who spend $70 \%$ of their time form filling, and only $30 \%$ on giving medical care. Little time is left for counseling and health promotion and women are forced to wait for long periods" (practising midwives group three).

Being able to spend time with the woman was considered the most important factor that influenced the quality of midwifery care. Participants reported that midwives routinely undertook a large number of non-midwifery duties, including clerical and housekeeping tasks that are an ineffective and inefficient use of their time and skills. Because of the burden of non-midwifery duties, midwives felt that they were unable to effectively undertake the role for which they were trained and employed. The short time that midwives spent with women did not allow them to develop a positive relationship. The midwife was always hurried, which did not allow women to feel that they could explore issues that were important to them. Unreasonable workloads and inflexibility in practice meant midwifery care was not responsive to the needs of women and was impersonal. Participants reported that they were frustrated as they were not able to work as they wanted. Midwifery educators considered that as a result to the lack of job descriptions which lead to blurring of professional roles, as midwifery educators said:

"The job description, which issued by the government, are not clear enough and related to administrative role rather than technical role" (midwifery educators). 
Participants identified that the most obvious means of reducing workload of practitioners was to ensure that staffing levels are adequate. This included administrative staff to reduce the paperwork burden. Participants asserted that midwives should spend most of their time involved in the direct care of the mother and baby. This was underpinned by the argument that qualified professionals are expensive to educate and employ, and that it makes little sense for them to be undertaking tasks that could be carried out by less expensive non-professional staff. The introduction of a midwifery assistant was seen as an option to help alleviate the increased non-midwifery workload of qualified staff. The role of a midwifery assistant would be able to help free the midwife from non-midwifery tasks.

\section{Medical domination of health services}

Participants highlighted issues relating to the medical domination of the health system. Opportunities for midwives to practise across the spectrum of maternity care varied according to how the services were organised and the prevailing philosophy of care. For example, a 'medical' model of care resulted in midwives experiencing a reduction in their autonomy in decision-making. This had the effect of midwives feeling that they were losing their skills and confidence in providing antenatal, intrapartum as well as postnatal care. This creates a problem as there is need for midwives to be confident in their knowledge base. Practising midwives as well as midwifery educators reported that:

"There is no ability to practice in the community due to lack of support and the medical dominance of all aspects of maternity care; we need facilitative policy that allows the midwife to practice as a PHC provider" (practising midwives - Group One).

"We agree that midwives should be able to take all responsibility for the care of healthy women. They should be autonomous and be very well trained ((midwifery educators).

Participants were concerned that they were required to value the medical model. There seemed to be a lack of midwifery leaders (in hierarchy as well as in knowledge) who could communicate directly with other senior staff. Participants reported that midwifery leadership would have positive benefits including increased confidence and improved interpersonal skills. Consequently, midwives felt that they could then 
work according to women's needs and new midwifery models could emerge. For example:

"If we agree that the pregnant woman is called a client not a patient, this means she must have more than one model to be able to choose the model they would prefer" (practising midwives - Group One).

\section{Midwifery Education}

Many participants referred to issues related to midwifery education when identifying barriers to midwifery service provision. Participants asked for direct entry midwifery programs ${ }^{1}$ to be improved and the length and quality of the program extended. Midwifery educators illustrated this by saying:

"Bachelor degree should be [the] minimum level of education for quality and to provide opportunities for higher education” (midwifery educators).

The other major educational issues identified by participants centred on the quality of clinical placements, the competency of the educators, the level of supervision of midwifery students and the lack of exposure to a full range of midwifery practice skills. It was felt that students did not have exposure to practice models other than those in a medicalised system. Participants stated that nurse educators were often inappropriate for midwives and the current route of educational and training criteria failed to give midwives a career path. The criteria that were applied which emerged from nursing thus difficult to apply to midwives. Midwifery educators reported:

"The profession needs international interchange to help improve quality of services as well as education" (midwifery educators).

Many participants said they were unable to access ongoing education and saw this as a major barrier to feeling confident to contribute effectively and safely to maternity service provision. Practising midwives said:

${ }^{1}$ Direct Entry Midwifery (DEM) refers to the direct entry into midwifery undergraduate education, or Bachelor of midwifery, without the requirement of a prerequisite undergraduate nursing program 
"As in nursing, [the] $\mathrm{MOH}$ must support and sponsor midwifery students and help with the cost in [a] bridging program to upgrade midwives" (practising midwives from group three).

\section{Discussion}

In this study, 91\% of those midwives invited participated. This indicated a high level of cooperation and interest in the topic from managers and leaders in the hospitals, as well as from midwives themselves. The reasons for the high participation rate may be due to midwives in this part of the world rarely refusing to participate if they know their answers will be handled confidentially, or it could relate to a cultural tendency to comply with requests. The researcher who approached the midwives was careful to explain the benefits of their participation in the study and assured them that confidentiality would be maintained. Furthermore, it appeared that many midwives did not have opportunities to participate in activities such as this research provided, due to their workload. Receiving a free day to express their feelings and sharing with colleagues acted as an incentive for participation in the study.

This study was only conducted in the government sector and with a relatively small sample, so it does not necessarily reflect midwives' perceptions and experiences throughout Jordan. The findings could be applied to the public sector, with a strong recommendation to conduct the study in other health sectors of Jordan.

The results from this research not only shows the magnitude to which the midwives' sampled are afflicted by perception and experience, but also supports previous findings from other studies in other countries (Brodie, 2002). For example, this study showed that professional midwives felt that they were not known or trusted by the community or the other professions. Midwives identified the need for education of medical personnel and the public regarding the scope of practice of midwives. Wagner (1998) noted that there is much ignorance and misunderstanding among the public and health professionals about the value of midwives in modern maternity services. Lack of reward is an increasing source of frustration in other countries (Ball et al., 2003) and contributes to role disengagement (Demerouti et al., 2000). 
The findings suggest that the medical model and non-evidence-based approaches predominate in the health services in Jordan. Khresheh et al. (2009) similarly asserted that many of clinical performance at maternity hospitals differ from evidence of best practice. The medical model, as Meleis and Im (2002) described, fosters women's dependency on physicians, consequently transferring control to physicians. In other words, the medical model promotes medicalisation of women's normal health-illness experiences and tends to make women feel helpless promoting a loss of control over their own ways of managing these health and illness experiences. Waldenstrom (1997) suggested that to define midwifery's area of practice, there should be a focus on evidence-based practice. Rejection of medicalised childbirth and a growing interest in the empowerment of women during childbirth has led to a renaissance of consumer demand for midwifery in other countries around the world (Mottl-Santiago, 2002).

Having sufficient time to provide quality care was an important concern for many participants. Many midwives in Jordan reported workloads of one midwife to 20 women and from experience, one midwife to around five women in labour. Recommendations from Scotland and elsewhere describe workloads of 'one midwife to one woman during labour and childbirth' and one midwife to four women when not in labour (Tucker et al., 2003). These ratios appear hard to reach in Jordan. Littlewood and Parker (1992) identified that lack of time is an important constraint to implementing primary health care properly in nursing. This lack of time was also evident in the Jordan and is likely to impact on nurses and midwives.

Employing an unqualified worker to assist the midwife is not a totally new phenomenon in Jordan. Traditionally, auxiliaries and receptionists/clerks have worked in maternity services and have been given a variety of titles to reflect the diversity of their roles (Hijazi and Al-Ma'aitah, 1999). Francomb (1997) identified tasks that could be delegated appropriately to assistants in maternity services in the United Kingdom (UK). These were categorised as housekeeping, hotel duties, and clerical duties. However, in Jordan, little guidance exists on the development of such a role and no distinction made between delegation to a midwife or an unqualified assistant. 
Participants in this study recognised the limits of their qualification and saw that as a problem. They wanted a baccalaureate as a minimum entry level of education to ensure quality. This has been reported in two previous studies in Jordan (Adams, 1994; Haddad and Umlauf, 1998). These studies revealed that midwifery programs are the weakest and narrowest form of entry into practice in Jordan. Graduates frequently lack preparation in areas such as health promotion and primary health care especially in community settings. Participants undervalued midwifery training in Jordan, although the graduates are now expected to perform important roles in primary health care services. This finding is similar to that described by Haddad and Umlauf (1998, p.527) who said "midwives [in Jordan] are not as well educated as midwives in developed countries”.

Practising midwives and midwifery educators raised issues about lack of access to educational opportunities because there were no higher degree programs. However, other factors severely affected their ability to take up these opportunities, most notably lack of finance, time and relief staff. Similar concerns were also reported by Abu-Luban (1990), who asserted that many Jordanian midwives are not competent to perform more advanced primary health care tasks which highlights the little progress made in the last 15 years. One reason might be due to a lack of clarity in job descriptions and the few resources for primary health care activities.

\section{Conclusion}

This study has shown that midwives recognised that developing their role within a PHC strategy will have benefits to themselves, their profession and the women and babies they care for. There are barriers that prevent midwives from undertaking this role. The major barriers were a lack of professional recognition; medical domination of health services; poor societal image; high levels of stress and workload; and problems with the quality of midwifery education. The lack of basic education and the low status of midwifery as a profession is problematic. Consequently, empowerment of midwives has been affected. It would appear from this study that an important consideration for midwives in their acceptance of new roles would be the implementation of strategies to overcome these barriers. The links between midwifery 
recognition, regulation and education were defined as areas to be focused upon and as a pathway towards change. 


\section{References}

Abu-Luban, A.,1990. National survey of health team job description and roles in primary health care centers. Jordan: Ministry of Health.

Adams, C., 1994. Midwifery in Jordan. Journal of Nurse Midwifery 39(3), 161-165.

Ball, J., Bennett, B., Washbrook, M., Webster, F., 2003. Factors affecting staffing ratios. British Journal of Midwifery 11(6), 357-361.

Brodie, P., 2002. Addressing the barriers to midwifery: Australian midwives speak out. Australian Journal of Midwifery 15(3), 5-14.

Briggs, C., Capdegelle, P., Garner, P., 2005. Strategies for integrating primary health services in middle- and low-income countries [Systematic Review]. Cochrane Database of Systematic Reviews 1, 3.

Carr, W., Kemmis, S.,1986. Becoming Critical: Knowing Through Action Research, Knowledge and Action Research. Victoria, Australia: Deakin University.

Cornwall, A., Jewkes, R.,1995. What is participatory research? Social Science Medical Journal 41(12), 1667-1676.

Demerouti, E., Bakker, A., Nachreiner, F., Schaufeli, W.,2000. A model of burnout and life satisfaction amongst nurses. Journal of Advanced Nursing 32(2), 454464.

Francomb, H., 1997. Do we need HCA's in the maternity services? British Journal of Midwifery 5, 672-679.

Haddad, L., Umlauf, M., 1998. Views of health promotion among primary health care nurses and midwives in Jordan. Health Care for Women International 19(6), 515-528.

Hijazi, S., Al-Ma'aitah, R.,1999. Public service reforms and their impact on health sector personnel in Jordan. Jordan: International Labour Office and World Health Organisation.

Hoskins, C., Mariano, C. (Eds)., 2004. Research In Nursing and Health: Understanding And Using Quantitative And Qualitative Methods. New York, NY: Springer Pub. Co.

Homer CSE, Passant L, Kildea S, Pincombe J, Thorogood C., Leap, N, Brodie, PM., 2007.The development of national competency standards for the midwife in Australia. Midwifery 23 (4): 350-360.

Hughes, I., Goolagong, P., Khavarpour, F., Russell, C.,1994. Action Research. Paper was presented to the Second Healing Our Spirit Worldwide Conference in Sydney, $14 \quad$ November.

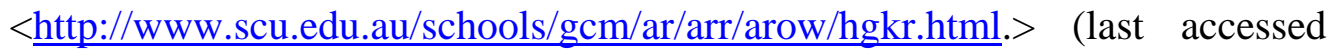
02.02.2004).

Jordan National Population Commission.,2000. Analysis of policy and legal barriers to improved reproductive health services in Jordan report. Amman-Jordan: The policy project.

Khresheh, R., Homer, C., Barclay, L., 2009. A comparison of labour and birth outcomes in Jordan with WHO guidelines: a descriptive study using a new birth record. Midwifery 25(6), 11-18.

Littlewood, J., Parker, I.,1992. Community nurses' attitudes of health promotion in one regional health authority. Health Education Journal 51(2), 87-89.

Meleis, A., Im, E., 2002. Grandmothers and women's health: from fragmentation to coherence. Health Care of Women International 23(2), 207-224. 
Meyer, J., 1993. New paradigm research in practice: the trials and tribulations of action research. Journal of Advanced Nursing 18(7), 1066-1072.

Mottl-Santiago, J., 2002. Women's public health policy in the 21st century. Journal of Midwifery and Women's Health 47(4), 228-238.

Noffke, S., Stevenson, R. (Eds), 1995. Educational Action Research: Becoming Practically Critical. New York: Teachers College Press.

Osmond, J.,Dar lington, Y., 2005. Reflective analysis: Techniques for facilitating reflection. Australian Social Work 58(1), 3-14.

Petro-Nustas, W., 2001. Factors associated with mammography utilization among Jordanian women. Journal of Transcultural Nursing 12(4), 284-291.

Polit, D., Beck, C., 2004. Nursing Research: principles and methods. Philadelphia; London: Lippincott Williams and Wilkins.

Rearick, M., Feldman, A., 1999. Orientations, product, reflections: A framework for understanding action research. Teaching and Teacher Education 15(4), 333349.

Shah, M.A., King, T.L., 2006. Competent Health Services for Women. Journal of Midwifery and Women's Health 51 (3): 139-140.

Sleeples, C., 2004. Using Action-Oriented Or Participatory Research Methods For Research on Networked Learning. <http://www.shef.ac.uk/nlc2004/Proceedings/Symposia/Symposium4/steeples. $>$ (last accessed 20.07.2005)

Sohng, S.,1995. Participatory research and community organising. Working paper presented at the New Social Movement and Community Organising Conference. <http://wwwinterweb.tech.com/nsmnet/docs/sohng.htm> (last accessed 11.08.2003)

Tucker, J., Parry, G., Penney, G., Page, M., Hundley, V., 2003. Is midwife workload associated with quality of process of care (continuous electronic fetal monitoring [CEFM]) and neonatal outcome indicators? A prospective study in consultant-led labour wards in Scotland. Paediatric and Perinatal Epidemiology 17(4), 369-377.

Wagner, M., 1998. Midwifery in the industrialized world. Journal of the Society of Obstetricians and Gynaecologicals of Canada 20(13), 1225-1234.

Waldenstrom, U., 1997. Challenges and Issues for Midwifery. Australian College of Midwives Incorporated Journal 10(3), 11-17.

Wass, A., 2000. Promoting Health: The Primary Health Care Approach. London: Harcourt Health Sciences Company.

WHO., 1978. Primary health Care: report of the International Conference of Primary health care. Alma Ata, USSR, Geneva, Switzerland. 